\title{
Exploring the relationship between high involvement work practices, work demands and employees' attitudes and behaviors
}

\author{
Panagiotis Kloutsiniotis
}

Purpose: The present study follows the conflicting outcomes perspective of the Human Resources Management (HRM) literature and examines the impact of employees' perceptions of High Performance Work Systems (HPWS) on their attitudes and behaviors, measured by job satisfaction and affective commitment. Moreover, this study hypothesizes that job responsibility and work overload mediate the relationship between high performance work practices and employees' attitudes. Last but not least, this survey follows the most recent development in the HRM literature and calculates HPWS as both a system and as subsets of HRM practices.

Design/methodology/approach: For the needs of this study, Partial Least Squares (PLS) Structural Equation Modeling (SEM) was used on a sample of 340 front-line employees working in one Greek manufacturing company. The data reported are drawn from a survey conducted in Spring 2018.

Findings: The findings do not support the critical arguments towards HPWS, which suggest that these systems lead to work intensification through enhanced job demands. Indeed, the results show first that these high performance work practices have a negative impact on employees' work overload, which in turn reduces their job satisfaction and affective commitment. Moreover, the findings show that HPWS enhances employees' job responsibility, which in turn increases their job satisfaction and affective commitment. Hence, both job responsibility and work overload mediate the relationship between HPWS and employees' attitudes and behaviors. Last but not least, of the three bundles of practices comprising the HPWS construct, the 'Abilities' one showed no statistical significance on the broader construct. Following the latter findings, we also ran the analysis separately for each bundle. These findings showed that the 'Abilities' bundle had no statistically significant effect on employees' job responsibility.

Practical implications: The findings suggest that HPWS creates positive effects on employees' well-being via two discrete mechanisms. First, by decreasing work overload, and secondly by increasing employees' job responsibility. Moreover, the findings underscore the detrimental impact that work overload may have on employees' job satisfaction and affective commitment, and highlights the need for management to pay specific attention in the conflicting outcomes perspective of HRM. Finally, the findings underscores to management the need to take into account not only the effects of high performance work practices as a system, but also as subsets of HRM practices.

Originality/value: This study follows the most recent developments in the Human Resource Management literature and examines the dark (negative) aspect of HPWS in the Greek manufacturing sector. In a nutshell, the theoretical and managerial implications drawn from this study improve our understanding of how HPWS influences employees' attitudes and behaviors. 